\title{
EVALUATION OF THE AARP DRIVER SAFETY PROGRAM IN FLORIDA
}

\author{
Gerald McGwin, Jr. and Cynthia Owsley \\ Departments of Ophthalmology and Epidemiology, \\ University of Alabama at Birmingham \\ Birmingham, AL USA \\ E-mail:mcgwin@uab.edu
}

PAPER NOT AVAILABLE

\begin{abstract}
\section{OBJECTIVES}

Older drivers are the fastest growing group of drivers on the road in the United States, both in terms of the number of drivers and annual mileage; also, per-mile-driven, this group has a crash rate nearly equivalent to that of younger drivers. One approach to reducing crash risk in this population has been educational programs aimed at improving driving skills and/or changing driving behaviors. This retrospective cohort study describes the impact of the AARP Driver Safety Program (DSP) in Florida on motor vehicle collisions and violations.
\end{abstract}

\section{METHODS}

Information on individuals who participated in the AARP DSP in the state of Florida in 2001 and 2002 was provided by the AARP. The Florida Department of Highway Safety and Motor Vehicles (FDHSMV) provided licensure information on all licensed drivers who were aged 60 and older as of January 1, 2001. With respect to violations and collisions, information dating from the mid-1990s to early 2005 was provided.

A total of 232,192 unique records for DSP participants were available; however, of these, 38,321 records were excluded because they contained out-of-state, erroneous or missing driver's license numbers and therefore could not be matched to the data provided by the FDHSMV. Of the remaining 193,871 records with legitimate Florida driver's license numbers, a total of 140,282 $(72.4 \%)$ could be linked to the data provided by the FDHSMV.

Two separate analyses were conducted. The first analysis compared violation and collision rates for DSP participants before and after DSP participation. The second analysis compared violation and collision rates between DSP participants and DSP non-participants.

\section{RESULTS}

Overall, DSP participants experienced a 7\% statistically significant decrease in collisions (rate ratio [RR] 0.93, 95\% confidence interval [CI] 0.89-0.97); a similar decrease was observed for injury-related collisions (RR $0.92,95 \%$ CI $0.88-0.98$ ) but not for fatal collisions (RR 1.20, 95\% CI 0.74-1.94). The decline in the overall collision rate can be attributed to a decline in not-at- 
fault collisions (RR 0.85, 95\% CI 0.80-0.91); there was no change in at-fault collisions (RR 1.00, 95\% CI 0.94-1.07).

Following DSP participation, violation rates significantly decreased $15 \%$ (RR $0.85,95 \%$ CI 0.83-0.87). This decrease was observed for all types of violations with the exception of failure to yield, improper turning and improper lane changing, all of which showed no change, and careless driving, which showed a statistically significant $11 \%$ increase (RR 1.11, 95\% CI 1.031.20).

Prior to DSP participation, those who participated in the DSP had a significantly higher collision rate (RR 1.11, 95\% CI 1.07-1.14) compared to DSP non-participants, independent of age, gender, and race. This significantly elevated rate was consistent across all types of collisions. Following DSP participation, the overall collision rate for DSP participants was higher than that of non-participants (RR 1.21, 95\% CI 1.17-1.25), though there was no difference for injurious or fatal collisions. The RR for not-at-fault and at-fault collisions indicated a lower collision rate for participants compared to non-participants.

With respect to violations, prior to DSP participation, those who ultimately took part in the DSP had a lower violation rate (RR $0.96,95 \%$ CI $0.95-0.98$ ), however, this was mostly attributable to seat belt usage (RR 0.64, 95\% CI 0.61-0.68). For all other types of violations, DSP participants had higher rates than the rest of the population. After DSP participation, participants had a higher violation rate compared to non-participants (RR 1.09, 95\% CI 1.08-1.11), as well as elevated rates for most types of violations. There was no difference for failure to obey traffic signals, careless/improper driving, or improper backing. DSP participants had a lower rate of seat belt violations.

\section{CONCLUSIONS}

The results of this analysis suggest that though individuals who participated in the DSP had an overall reduction in collision rates, this reduction was attributable to not-at-fault collisions. This likely indicates that DSP participants modified their driving habits following the program (e.g., reduced their exposure) and did not necessarily improve their driving skills. There was also a reduction is some types of collisions, as well as an increase in careless driving-related offenses. These reductions may reflect a greater adherence to traffic laws or may simply reflect a reduction in driving brought about by changes in driving habits.

Compared to similarly aged Florida drivers who did not participate in the DSP, participants had higher collision rates prior to the DSP. After DSP participation, the observed differences between participants and non-participants were either diminished or inverted such that participants had lower rates compared to non-participants. Participants had an overall lower violation rate prior to the DSP but for the most common types of violations they had elevated rates. Following participation, DSP participants had a higher crash rate compared to the rest of the population. For specific types of violations the elevated rates persisted but were diminished in magnitude. 